\title{
Heterogeneous sorption of radionuclides predicted by crystal surface
}

\section{nanoroughness}

3 Tao Yuan*1, Stefan Schymura ${ }^{1}$, Till Bollermann ${ }^{1}$, Konrad Molodtsov ${ }^{1}$, Paul Chekhonin ${ }^{1}$, Moritz Schmidt ${ }^{1}$,

$4 \quad$ Thorsten Stumpf ${ }^{1}$, and Cornelius Fischer ${ }^{1}$

5 Institute of Resource Ecology, Helmholtz-Zentrum Dresden-Rossendorf (HZDR), Dresden, Saxony, 01328,

6 Germany

$7 \quad{ }^{*}$ Corresponding Author: t.yuan@hzdr.de

8 The supporting information includes 5 tables and 10 figures on 11 pages. 
Table S1. Field of views (FOV) and resolutions in x,y, and z for the used Nikon interferometric objectives

Table S2. Results of Student t-tests $(p=0.05)$ for europium sorption on different grains with indicated effect sizes according to Cohen's $\mathrm{d}^{1}(\square:$ no statistical significance, $\square:$ small effect $(\mathrm{d} \geq 0.2), \square:$ medium effect $(\mathrm{d} \geq$ $0.5)$, $\square:$ large effect $(\mathrm{d} \geq 0.8)$ ).

Table S3. Results of Student t-tests $(p=0.05)$ for europium sorption on different groups of grains (top) and grains A, I, D, B, used for detailed topography analysis with indicated effect sizes according to Cohen's $\mathrm{d}^{1}$ ( $\square:$ no statistical significance, $\square:$ small effect $(\mathrm{d} \geq 0.2), \quad$ : medium effect $(\mathrm{d} \geq 0.5), \square:$ large effect $(\mathrm{d} \geq 0.8))$

Table S4. Reactions and Reaction Parameters in SCM

Table S5. Determined lifetimes of selected data points with the correlated amount of remaining water molecules along with the species distribution factors

\section{List of Figures}

Figure S1. Europium sorption on polycrystalline calcite grains A-J, ordered by increasing europium sorption (top, left). EBSD map of the investigated surface with grains A-J and their respective crystal orientations (top, right). Europium sorption on groupings of calcite grains AF, IJHG, EDC, and B, with no statistically significant differences in europium sorption (bottom, left). Europium sorption on grains A, I, D, and B used for detailed analysis (bottom, right). Error bars indicate one standard deviation (SD).

Figure S2. Correlation of the sorption capacity with the Sq value averaged over the respective $2.5 \times 2.5 \mu \mathrm{m}$ subpixels in the ranges 0-5 nm (top, left), 5-10 nm (top, right), 10-50 nm (bottom, left) and $>50 \mathrm{~nm}$ (bottom, right).

Figure S3. Comparison of sorption efficiency between SCM results with published experimental data

Figure S4. Dependency of $\alpha$ on the FOV of the Sq data. As the FOV of the Sq data increases, $\alpha$ decreases according to a power law. The $\alpha$ for the predictive modeling was set to $\alpha=0.03$ to adapt the model for the higher resolution of the topographical data.

Figure S5. The scatter plots of measured sorbed Eu(III) TRLFS signal versus $\mathrm{S}_{\mathrm{dr}}$. The $\mathrm{S}_{\mathrm{dr}}$ values of each subpixel are calculated by the software SPIP (version: 6.7.9) with measured surface topographies.

Figure S6. Effect of FOV convergence from $20 \times 20 \mu \mathrm{m}^{2}$ to $2.5 \times 2.5 \mu \mathrm{m}^{2}$ of the Sq calculation on the coefficient of determination of the linear fit of the correlation.

Figure S7. Illustration of the narrowing of the data spread when correcting for exaggerated Eu sorption and Sq values.

Figure S8. The scatter plots of the calculated surface area ratio $\left(\mathrm{S}_{\mathrm{dr}}\right)$ versus calculated average Sq. Sq values are calculated at a $2.5 \times 2.5 \mu \mathrm{m}^{2}$ FOV. Strong linear correlation can be observed with $\mathrm{R}^{2}=0.82$

Figure S9. Comparison of sorption efficiency between two SCM results. In SCM 1, we change SSA and fix SSD. In SCM 2, we change SSD and fix SSA. SSA $=0.262 \mathrm{~m}^{2} / \mathrm{g}$ and SSD $=5 \mathrm{sites} / \mathrm{nm}^{2}$ are the reference values to match the data from literature. The a in horizontal axis is a relative value with respect to the reference values for each case.

Figure S10. The ratio of sorbed $\mathrm{Eu}(\mathrm{III})$ by the $\mathrm{SCM}$ to $\mathrm{Eu}(\mathrm{III})$ by experiment versus $\alpha$. Points $\mathrm{A}(\alpha=0.0038)$ and $\mathrm{B}(\alpha=0.0075)$ are the two ratios for model validation in ROIs I\&II and III, respectively. 
76 Table S1. Field of views (FOV) and resolutions in $\mathrm{x}, \mathrm{y}$, and $\mathrm{z}$ for the used Nikon interferometric objectives

\begin{tabular}{|l|l|l|l|}
\hline Objective & DI 100x & DI 50x & DI 20x \\
\hline FOV $[\mu \mathrm{m}]$ & $170 \times 142$ & $340 \times 284$ & $850 \times 710$ \\
\hline Res $x y[\mu \mathrm{m}]$ & 0.2 & 0.28 & 0.69 \\
\hline Res $\mathrm{z}[\mathrm{nm}]$ & 1 & 1 & 1 \\
\hline
\end{tabular}
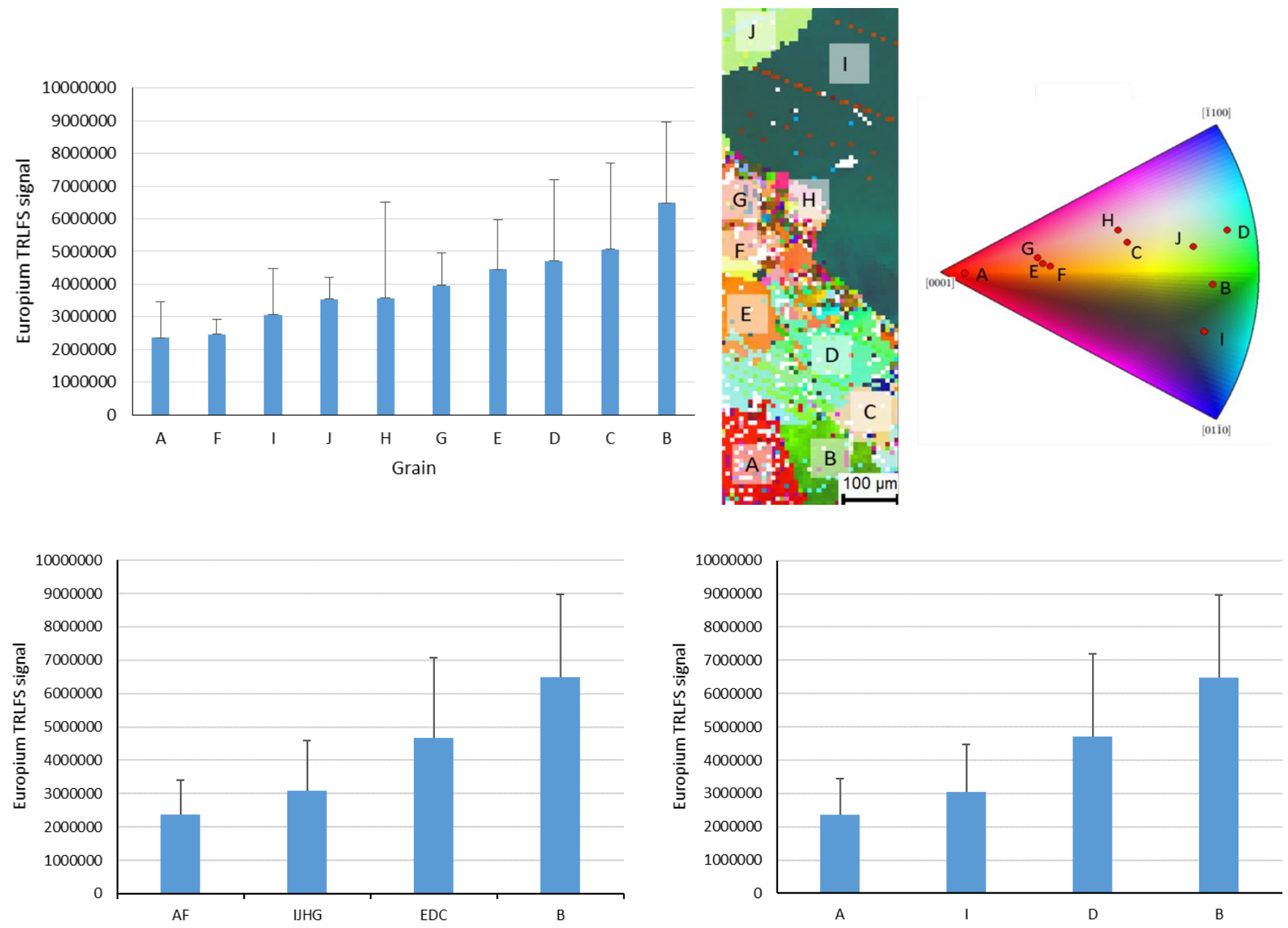

80 Figure S1. Europium sorption on polycrystalline calcite grains A-J, ordered by increasing europium sorption 81 (top, left). EBSD map of the investigated surface with grains A-J and their respective crystal orientations (top, 82 right). Europium sorption on groupings of calcite grains AF, IJHG, EDC, and B, with no statistically significant 83 differences in europium sorption (bottom, left). Europium sorption on grains A, I, D, and B used for detailed 84 analysis (bottom, right). Error bars indicate one standard deviation (SD). 
Table S2. Results of Student t-tests $(\mathrm{p}=0.05)$ for europium sorption on different grains with indicated effect sizes according to Cohen's $\mathrm{d}^{1}(\square:$ : no statistical significance, $\square:$ small effect $(\mathrm{d} \geq 0.2), \square$ : medium effect $(\mathrm{d} \geq 0.5), \square$ : $87 \quad$ large effect $(\mathrm{d} \geq 0.8))$.

\begin{tabular}{|l||r|r|r|r|r|r|r|r|r|r|}
\hline $\begin{array}{l}\text { Cohen's } \\
\text { d }\end{array}$ & A & B & C & D & E & F & G & H & I & J \\
\hline \hline A & & 0.81 & 0.71 & 0.64 & 0.67 & & 0.81 & 0.57 & 0.45 & 0.71 \\
\hline B & 0.81 & & 0.25 & 0.37 & 0.50 & 0.84 & 0.81 & 0.51 & 0.80 & 0.76 \\
\hline C & 0.71 & 0.25 & & & 0.25 & 0.70 & 0.55 & & 0.65 & 0.53 \\
\hline D & 0.64 & 0.37 & & & & 0.90 & 0.78 & & 0.57 & 1.10 \\
\hline E & 0.67 & 0.50 & 0.25 & & & 0.75 & 0.49 & & 0.60 & 0.46 \\
\hline F & & 0.84 & 0.70 & 0.90 & 0.75 & & 0.61 & 0.54 & 0.77 & 0.68 \\
\hline G & 0.81 & 0.81 & 0.55 & 0.78 & 0.49 & 0.61 & & & \\
\hline H & 0.57 & 0.51 & & & & 0.54 & & & \\
\hline I & 0.45 & 0.80 & 0.65 & 0.57 & 0.60 & 0.77 & & & \\
\hline J & 0.71 & 0.76 & 0.53 & 1.10 & 0.46 & 0.68 & & & \\
\hline
\end{tabular}

Table S3. Results of Student t-tests $(\mathrm{p}=0.05)$ for europium sorption on different groups of grains (top) and grains A, I, D, B, used for detailed topography analysis with indicated effect sizes according to Cohen's d $\mathrm{d}^{1}$ ( $\square:$ no statistical significance, $\square:$ small effect $(\mathrm{d} \geq 0.2)$, : medium effect $(\mathrm{d} \geq 0.5)$, $\square:$ large effect $(\mathrm{d} \geq 0.8))$

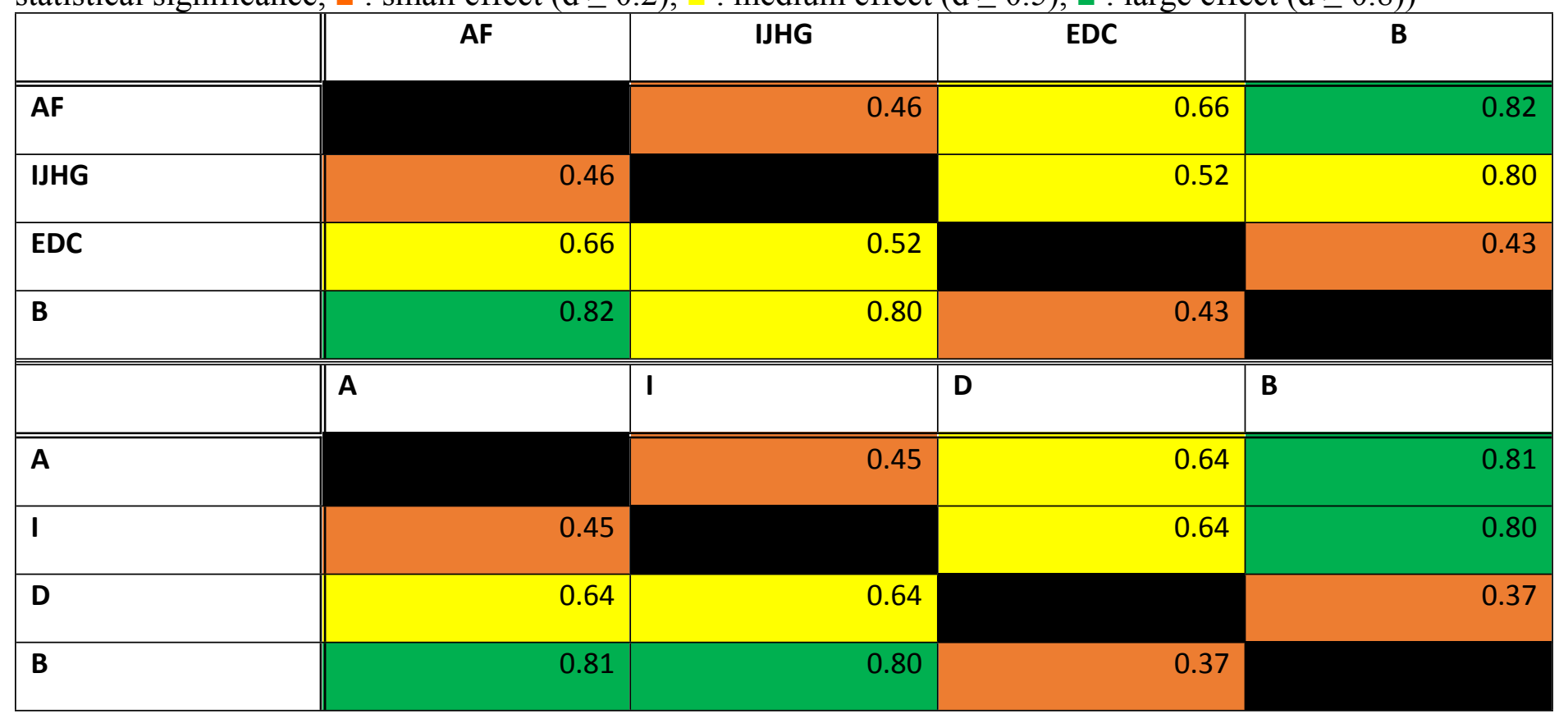


$\mathrm{Sq}(0-5 \mathrm{~nm})$
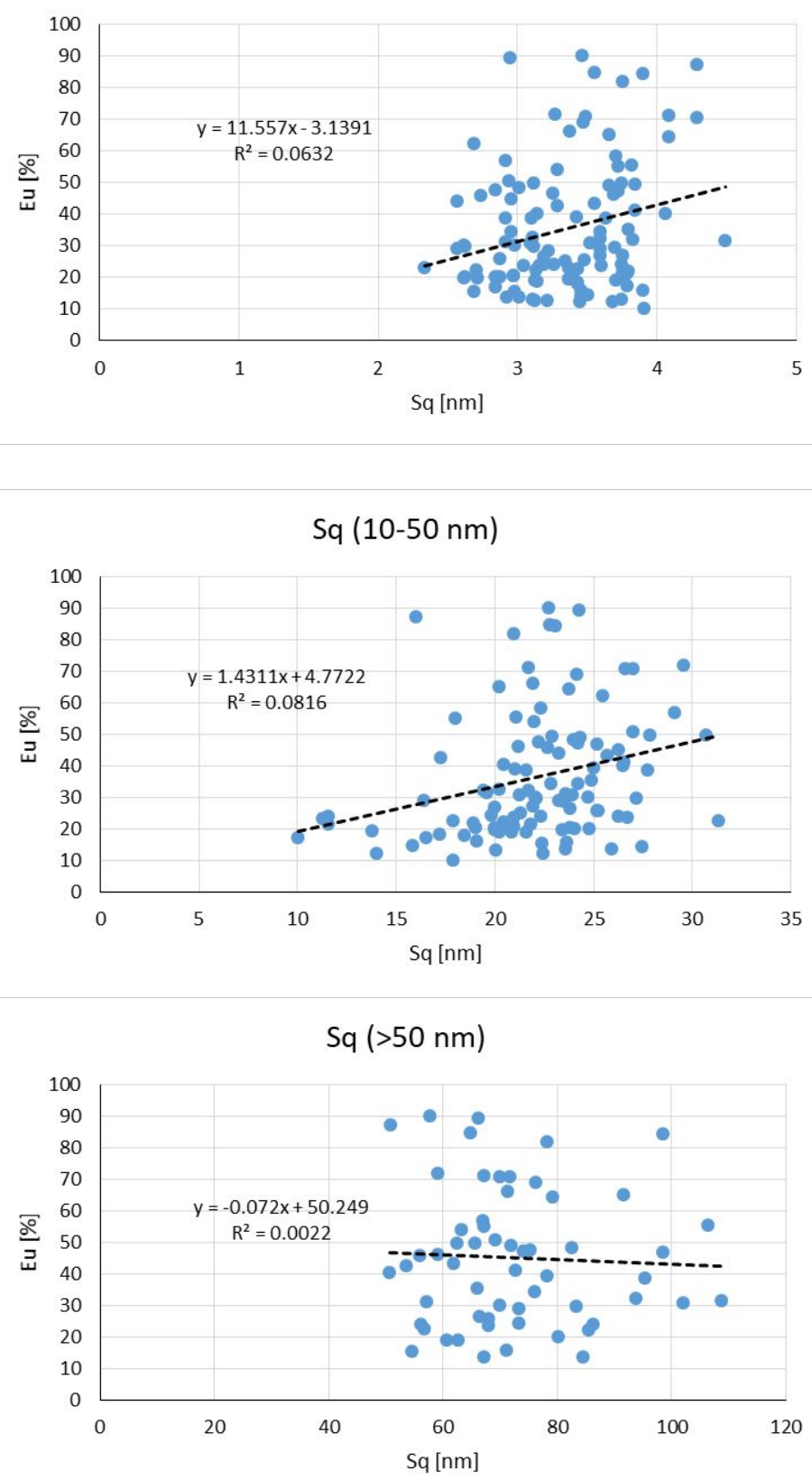

Figure S2. Correlation of the sorption capacity with the Sq value averaged over the respective $2.5 \times 2.5 \mu \mathrm{m}$ subpixels in the ranges 0-5 $\mathrm{nm}$ (top, left), 5-10 nm (top, right), 10-50 nm (bottom, left) and $>50 \mathrm{~nm}$ (bottom, right). 


\begin{tabular}{|c|c|}
\hline $\mathbf{E u}^{3+}+\mathrm{H}_{2} \mathbf{O} \rightleftharpoons \mathrm{EuOH}^{2+}+\mathrm{H}^{+}$ & $-7.9^{\mathrm{a}}$ \\
\hline $\mathrm{Eu}^{3+}+2 \mathrm{H}_{2} \mathrm{O} \rightleftharpoons \mathrm{Eu}(\mathrm{OH})^{+}{ }_{2}+2 \mathrm{H}^{+}$ & $-16.37^{a}$ \\
\hline $\mathrm{Eu}^{3+}+3 \mathrm{H}_{2} \mathrm{O} \rightleftharpoons \mathrm{Eu}(\mathrm{OH})_{3}+3 \mathrm{H}^{+}$ & $-25.41^{\mathrm{a}}$ \\
\hline $\mathrm{Eu}^{3+}+4 \mathrm{H}_{2} \mathrm{O} \rightleftharpoons \mathrm{Eu}(\mathrm{OH})^{-}{ }_{4}+4 \mathrm{H}^{+}$ & $-34.51^{\mathrm{a}}$ \\
\hline $\mathrm{Eu}^{3+}+\mathrm{HCO}_{3}^{-} \rightleftharpoons \mathrm{EuHCO}_{3}^{2+}$ & $2.10^{\mathrm{a}}$ \\
\hline $\mathrm{Eu}^{3+}+\mathrm{HCO}_{3}^{-} \rightleftharpoons \mathrm{EuCO}_{3}^{+}+\mathrm{H}^{+}$ & $-2.43^{\mathrm{a}}$ \\
\hline $\mathrm{Eu}^{3+}+2 \mathrm{HCO}_{3}^{-} \rightleftharpoons \mathrm{Eu}\left(\mathrm{CO}_{3}\right)_{2}^{-}+2 \mathrm{H}^{+}$ & $-7.76^{\mathrm{a}}$ \\
\hline $\mathrm{Eu}^{3+}+3 \mathrm{HCO}_{3}^{-} \rightleftharpoons \mathrm{Eu}\left(\mathrm{CO}_{3}\right)_{3}^{3-}+2 \mathrm{H}^{+}$ & $-18.49^{a}$ \\
\hline Surface complexation reactions & $\log (\mathrm{K})$ \\
\hline$\equiv \mathrm{K}_{\mathbf{1}} \mathbf{O H} \rightleftharpoons \equiv \mathrm{K}_{\mathbf{1}} \mathbf{O}^{-}+\mathrm{H}^{+}$ & $-5.10^{\mathrm{a}}$ \\
\hline$\equiv \mathbf{K}_{\mathbf{1}} \mathbf{O H}+\mathrm{Ca}^{2+} \rightleftharpoons \equiv \mathbf{K}_{\mathbf{1}} \mathbf{O C a}^{+}+\mathbf{H}^{+}$ & $-1.70^{\mathrm{a}}$ \\
\hline$\equiv \mathbf{K}_{2} \mathbf{O H} \rightleftharpoons \equiv \mathbf{K}_{2} \mathbf{O}^{-}+\mathbf{H}^{+}$ & $-12.0^{\mathrm{a}}$ \\
\hline$\equiv \mathbf{K}_{\mathbf{2}} \mathbf{O H}+\mathbf{H}^{+} \rightleftharpoons \equiv \mathbf{K}_{\mathbf{2}} \mathbf{O H}_{2}^{+}$ & $11.85^{\mathrm{a}}$ \\
\hline$\equiv \mathrm{K}_{2} \mathrm{OH}+\mathrm{HCO}_{3}^{-}+\mathrm{H}^{+} \rightleftharpoons \equiv \mathrm{K}_{2} \mathrm{HCO}_{3}+\mathrm{H}_{2} \mathbf{O}$ & $20.5^{\mathrm{b}}$ \\
\hline$\equiv \mathrm{K}_{2} \mathrm{OH}+\mathrm{HCO}_{3}^{-} \rightleftharpoons \equiv \mathrm{K}_{2} \mathrm{CO}_{3}^{-}+\mathrm{H}_{2} \mathrm{O}$ & $0.77^{b}$ \\
\hline$\equiv \mathrm{K}_{2} \mathrm{OH}+\mathrm{Eu}^{3+}+\mathrm{HCO}_{3}^{-} \rightleftharpoons \equiv \mathrm{K}_{2} \mathrm{OEuCO}_{3}+2 \mathrm{H}^{+}$ & $5.5^{\mathrm{b}}$ \\
\hline$\equiv \mathrm{K}_{2} \mathrm{OH}+\mathrm{Eu}^{3+}+2 \mathrm{HCO}_{3}^{-} \rightleftharpoons \equiv \mathrm{K}_{2} \mathrm{OEu}\left(\mathrm{CO}_{3}\right)_{2}^{2-}+3 \mathrm{H}^{+}$ & $4.6^{\mathrm{b}}$ \\
\hline
\end{tabular}




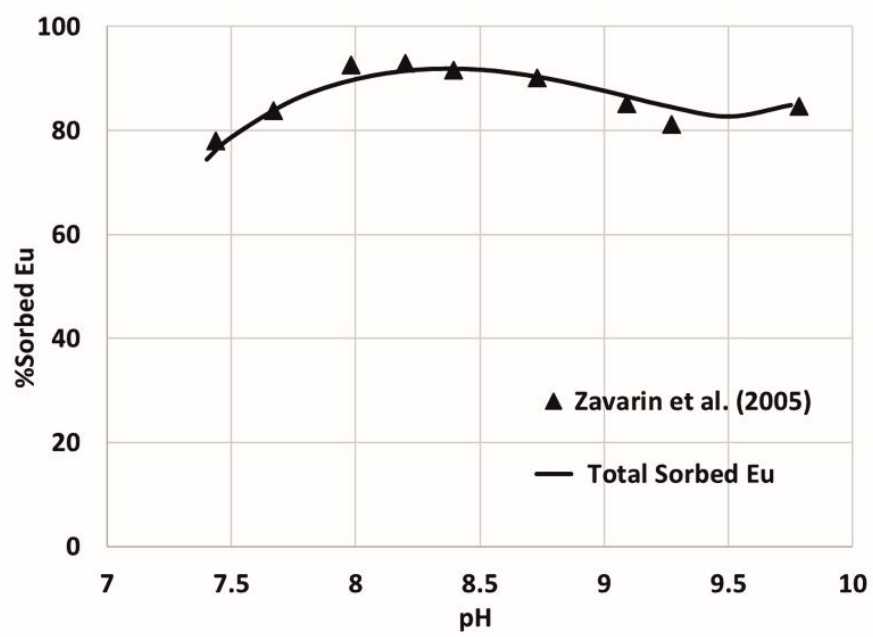

Figure S3. Comparison of sorption efficiency between SCM results with published experimental data

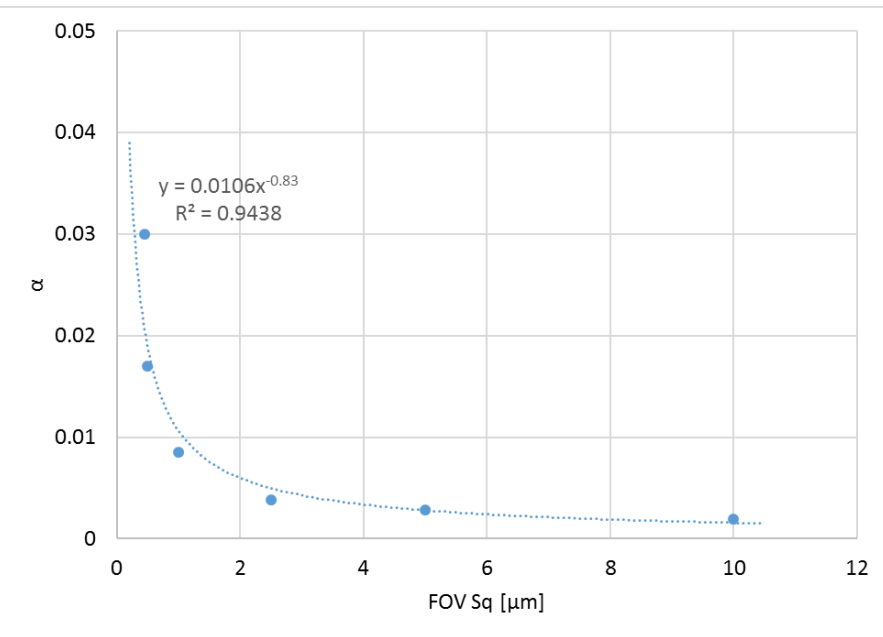

Figure S4. Dependency of $\alpha$ on the FOV of the Sq data. As the FOV of the Sq data increases, $\alpha$ decreases according to a power law. The $\alpha$ for the predictive modeling was set to $\alpha=0.03$ to adapt the model for the higher resolution of the topographical data.

The lifetime of $\mathrm{Eu}^{3+}$ is determined in several data points with high and low luminescence intensity to get an overview of the speciation in the whole ROI. The intensity-time-pattern is partly reconstructed with a monoexponential decay in the case of pixels with low luminescence intensity (purple hollow dots) resulting in a lifetime of around $2000 \mu \mathrm{s}$, which corresponds to 0.0 remaining water molecules in the first coordination sphere, 113 representing $\mathrm{Eu}^{3+}$ incorporated into the crystal lattice. The lifetime is lower than $3600 \mu \mathrm{s}$ reported in the literature ${ }^{2}$. 114 This discrepancy is most likely an artefact of the low luminescence intensity and/or minor contributions of 
115 adsorbed $\mathrm{Eu}^{3+}$. To measure such long lifetimes precisely high delays of up to $10 \mathrm{~ms}$ are needed, which results in

116 luminescence intensities below the detection limit. Therefore it can be assumed that those $2000 \mu$ s correspond to

117 the calcite incorporation species with $3600 \mu$ s mixed with very low parts of sorption. In some of the purple data 118 points surface sorption species can be determined with between 1.5 and 4.0 water molecules remaining. Due to 119 the low luminescence intensity, those lifetimes are once again affected by uncertainties. The blue data points show 120 a much more intense sorption species. The short lifetime has a mean value of 2.3 remaining water molecules with a fixed long lifetime of $3600 \mu \mathrm{s}$. The ratio between sorption (short) and incorporation (long) varies between 1:1 and $4: 1$.

Table S5. Determined lifetimes of selected data points with the correlated amount of remaining water molecules along with the species distribution factors

\begin{tabular}{|c|c|c|c|c|c|c|}
\hline \multirow{2}{*}{ Coordinates } & \multicolumn{3}{|c|}{ Short } & \multicolumn{2}{|c|}{ Long } & \multirow{2}{*}{$A_{s}: A_{l}$} \\
\hline & $\tau_{\mathrm{s}}[\mu \mathrm{s}]$ & $\mathrm{n}_{\mathrm{s}}\left(\mathrm{H}_{2} \mathrm{O}\right)$ & $\mathrm{A}_{\mathrm{S}}[\%]$ & $\tau_{l}[\mu \mathrm{s}]$ & $\mathrm{A}_{l}[\%]$ & \\
\hline $3 / 4$ (purple) & $233 \pm 37$ & $4.0 \pm 0.7$ & $51 \pm 4$ & 3600 & $49 \pm 2$ & 1.0 \\
\hline 14/7 (purple) & - & - & - & $2034 \pm 232$ & $100 \pm 0$ & - \\
\hline 10/8 (purple) & $479 \pm 220$ & $1.6 \pm 1.0$ & $33 \pm 6$ & 3600 & $67 \pm 6$ & 0.5 \\
\hline 3/22 (purple) & - & - & - & $2019 \pm 162$ & $100 \pm 0$ & - \\
\hline $5 / 30$ (purple) & - & - & - & $1972 \pm 159$ & $100 \pm 0$ & - \\
\hline 20/37 (blue) & $276 \pm 45$ & $3.3 \pm 0.6$ & $45 \pm 3$ & 3600 & $55 \pm 2$ & 0.8 \\
\hline 13/38 (blue) & $555 \pm 54$ & $1.3 \pm 0.2$ & $65 \pm 3$ & 3600 & $35 \pm 3$ & 1.9 \\
\hline 9/44 (blue) & $339 \pm 63$ & $2,5 \pm 0.6$ & $48 \pm 4$ & 3600 & $52 \pm 3$ & 0.9 \\
\hline 12/48 (blue) & $412 \pm 18$ & $2.0 \pm 0.1$ & $78 \pm 2$ & 3600 & $22 \pm 1$ & 3.6 \\
\hline
\end{tabular}




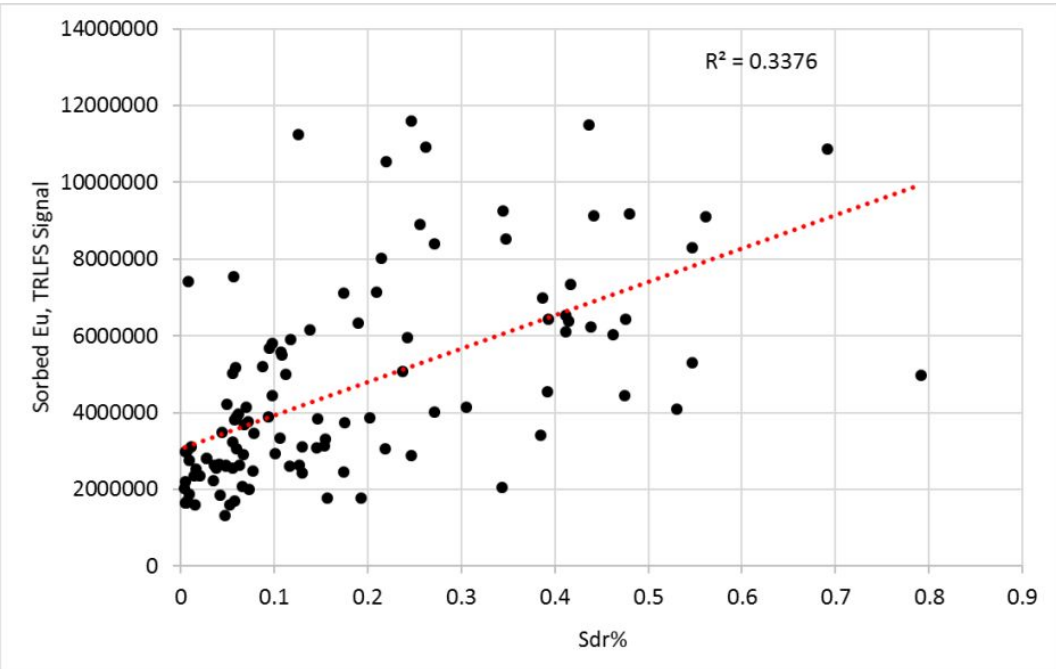

Figure S5. The scatter plots of measured sorbed Eu(III) TRLFS signal versus $S_{d r}$. The $S_{d r}$ values of each subpixel are calculated by the software SPIP (version: 6.7.9) with measured surface topographies.

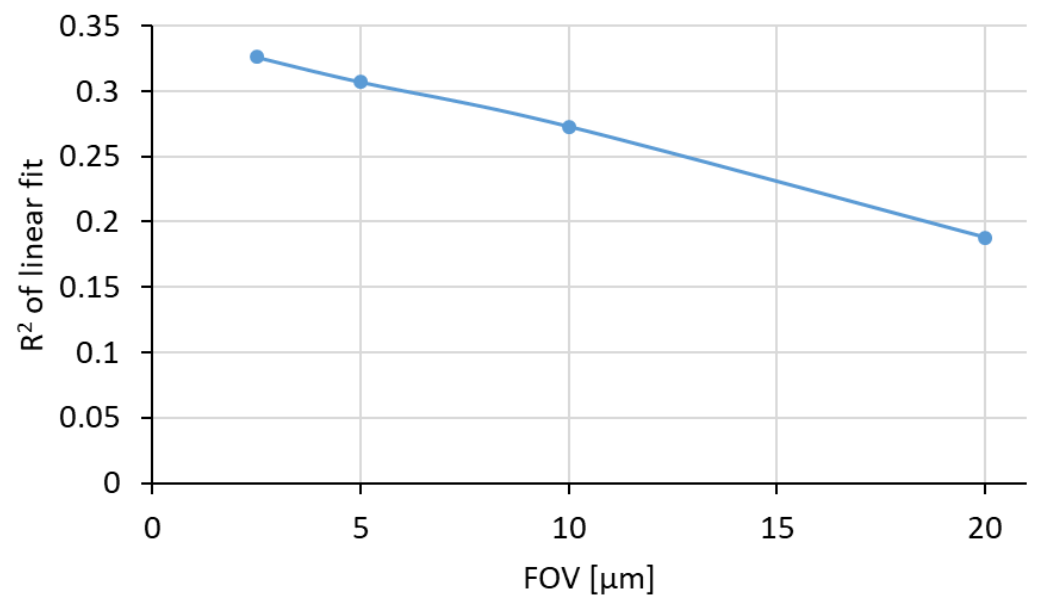

Figure S6. Effect of FOV convergence from $20 \times 20 \mu \mathrm{m}^{2}$ to $2.5 \times 2.5 \mu \mathrm{m}^{2}$ of the Sq calculation on the coefficient of determination of the linear fit of the correlation. 


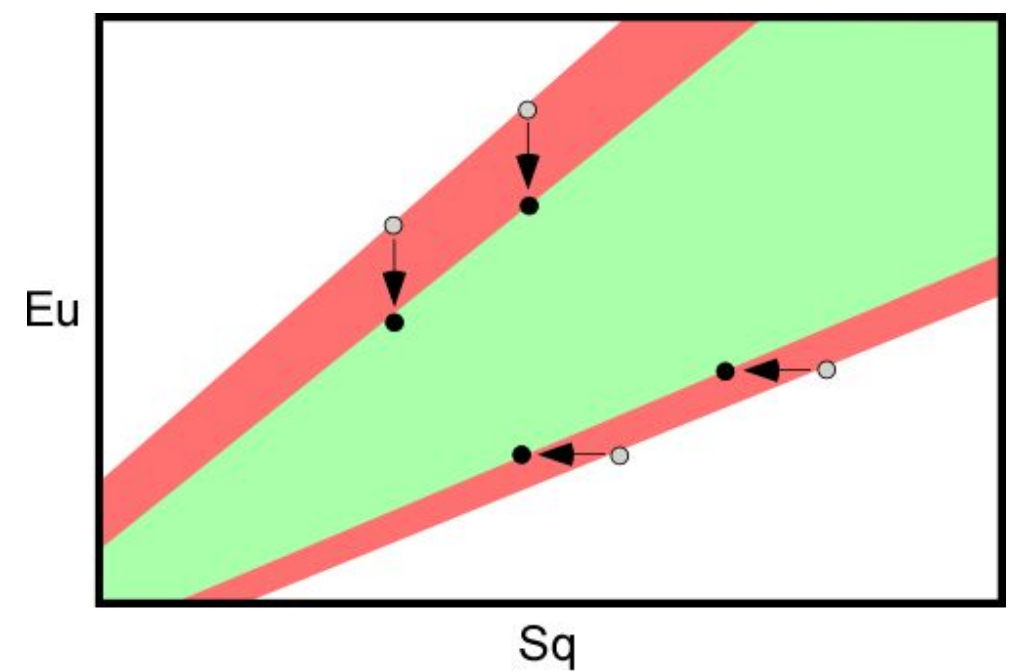

137 Figure S7. Illustration of the narrowing of the data spread when correcting for exaggerated Eu sorption and Sq values.

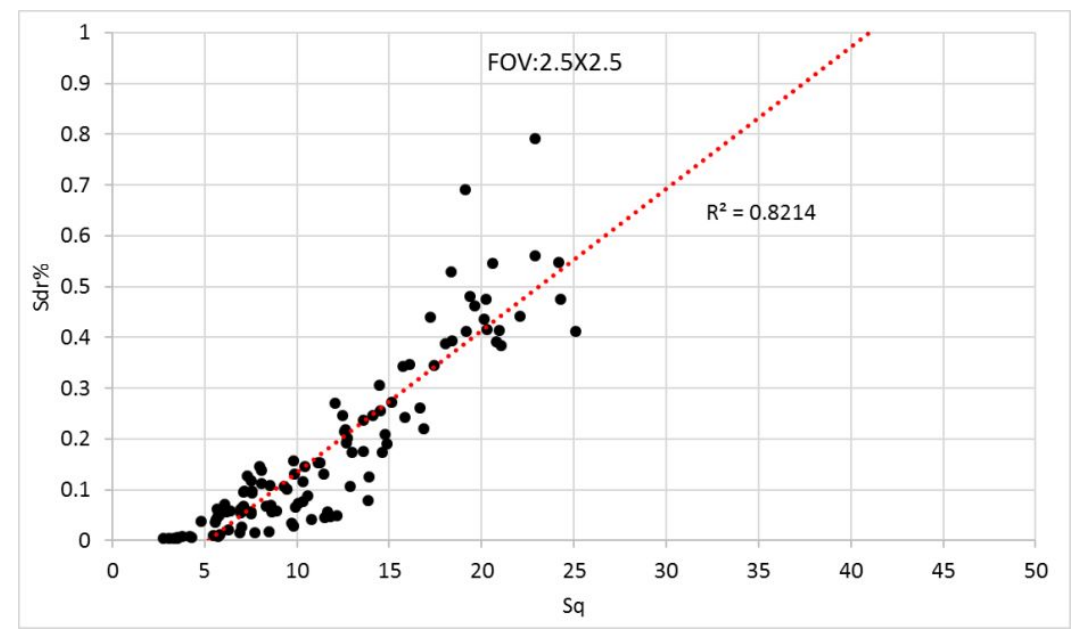

140 Figure S8. The scatter plots of the calculated surface area ratio $\left(\mathrm{S}_{\mathrm{dr}}\right)$ versus calculated average Sq. Sq values are 


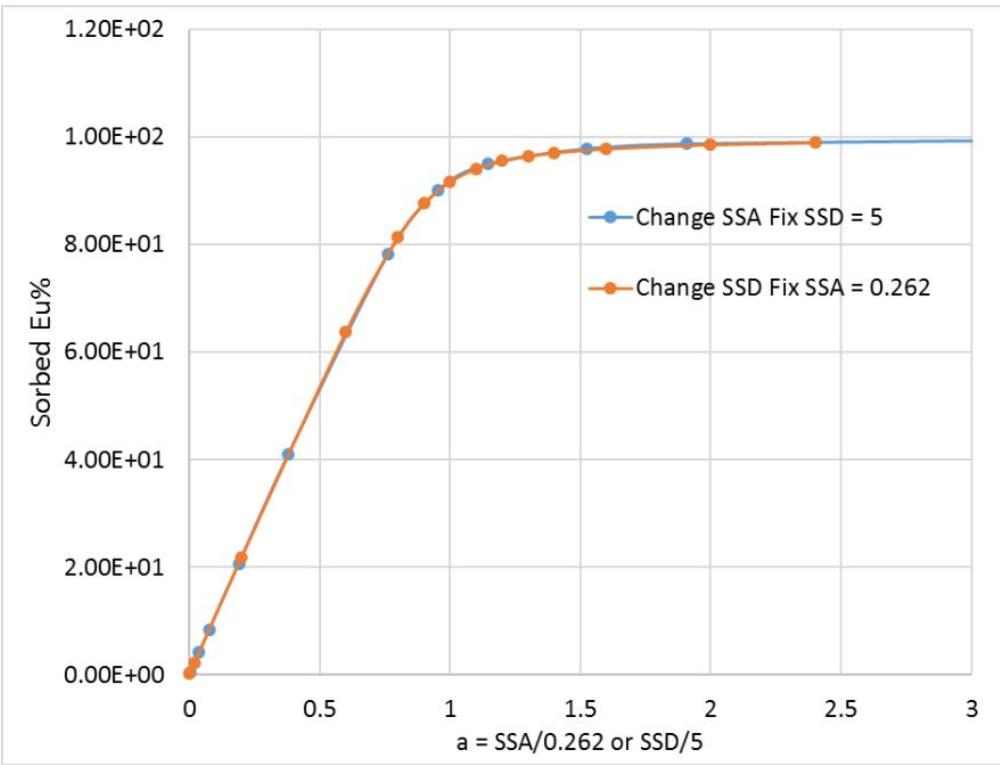

Figure S9. Comparison of sorption efficiency between two SCM results. In SCM 1, we change SSA and fix SSD. In SCM 2, we change SSD and fix SSA. SSA $=0.262 \mathrm{~m}^{2} / \mathrm{g}$ and $\mathrm{SSD}=5$ sites $/ \mathrm{nm}^{2}$ are the reference values to match the data from literature. The a in horizontal axis is a relative value with respect to the reference values for each case.

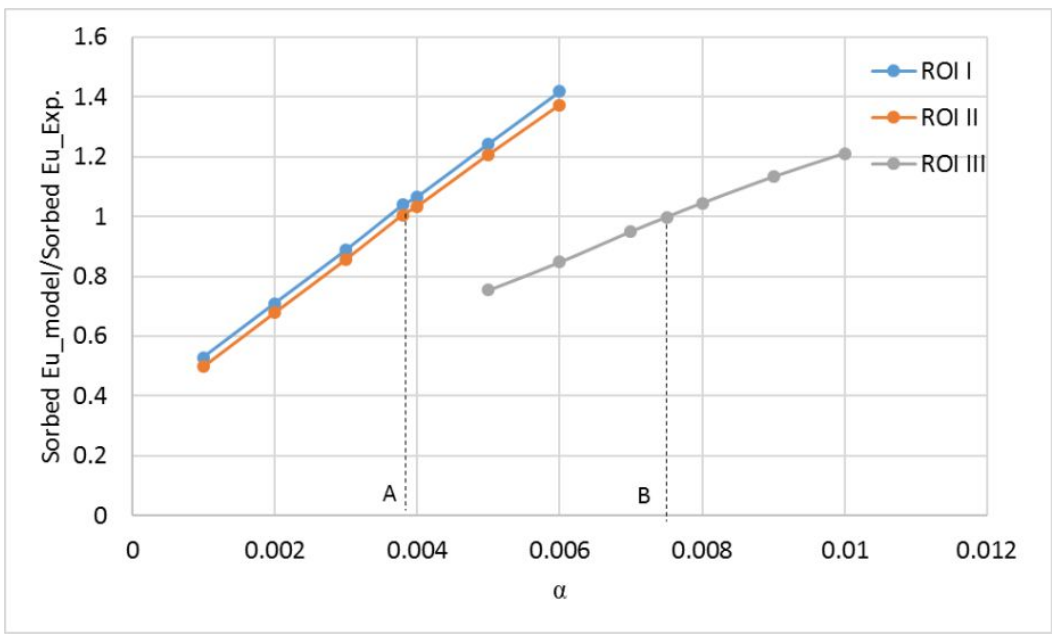

Figure S10. The ratio of sorbed $\mathrm{Eu}(\mathrm{III})$ by the $\mathrm{SCM}$ to $\mathrm{Eu}(\mathrm{III})$ by experiment versus $\alpha$. Points $\mathrm{A}(\alpha=0.0038)$ and $\mathrm{B}(\alpha=0.0075)$ are the two ratios for model validation in ROIs I\&II and III, respectively.

\section{References}

1. Sawilowsky, S. S., New Effect Size Rules of Thumb. Journal of Modern Applied Statistical Methods 2009, $8(2), 467-474$. solid solutions. Angew. Chem. Int. Ed. Engl. 2008, 47(31), 5846-50. 